\title{
Existence of three solutions for a Navier boundary value problem involving the $(p(x), q(x))$-biharmonic
}

\author{
Feng-Li Huang ${ }^{1}$, Guang-Sheng Chen ${ }^{2 *}$, Yu-Qi Niu ${ }^{3}$ and Ti Song ${ }^{4}$
}

\section{"Correspondence:}

cgswavelets@126.com

${ }^{2}$ Department of Construction and

Information Engineering, Guangxi

Modern Vocational Technology

College, Hechi, Guangxi 547000,

China

Full list of author information is

available at the end of the article

\begin{abstract}
In this paper, we study $(p(x), q(x))$-biharmonic systems with Navier boundary condition on a bounded domain and obtain three solutions under appropriate hypotheses. The technical approach is mainly based on the general three critical points theorem obtained by Ricceri.
\end{abstract}

Keywords: three solutions; $(p(x), q(x))$-biharmonic; Navier condition; Ricceri's three critical points theorem

\section{Introduction and main results}

In this paper, we consider the Navier boundary value problem involving the $(p(x), q(x))$ biharmonic systems

$$
\begin{cases}\Delta\left(|\Delta u|^{p(x)-2} \Delta u\right)=\lambda F_{u}(x, u, v)+\mu G_{u}(x, u, v), & \text { in } \Omega, \\ \Delta\left(|\Delta u|^{q(x)-2} \Delta u\right)=\lambda F_{v}(x, u, v)+\mu G_{v}(x, u, v), & \text { in } \Omega, \\ u=\Delta u=v=\Delta v=0, & \text { on } \partial \Omega,\end{cases}
$$

where $\lambda, \mu \in[0,+\infty), \Omega \subset R^{N}(N \geq 1)$ is a nonempty bounded open set with a boundary $\partial \Omega$ of class $C^{1}, F, G: \Omega \times R \times R \rightarrow R$ are functions such that $F(\cdot, s, t), G(\cdot, s, t)$ are measurable in $\Omega$ for all $(s, t) \in R \times R$ and $F(x, \cdot, \cdot)$ is $C^{1}$ in $R \times R$ for a.e. $x \in \Omega, F_{i}$ denotes the partial derivative of $F$ with respect to $i, i=u, v$, so does $G_{i}$. And $p, q \in C(\bar{\Omega}), 1<p^{-}=\inf _{x \in \bar{\Omega}} p(x) \leq$ $p^{+}=\sup _{x \in \bar{\Omega}} p(x)<+\infty, 1<q^{-}=\inf _{x \in \bar{\Omega}} q(x) \leq q^{+}=\sup _{x \in \bar{\Omega}} q(x)<+\infty$. Moreover,

$$
p^{*}(x)= \begin{cases}\frac{N p(x)}{N-p(x)} & \text { if } p(x)<N \\ \infty & \text { if } p(x) \geq N\end{cases}
$$

is the critical exponent just as in many papers. Obviously, $p(x)<p^{*}(x), q(x)<q^{*}(x)$ for all $x \in \Omega$.

In what follows, $E$ denotes the Cartesian product of two Sobolev spaces $W^{2, p(x)}(\Omega) \cap$ $W_{0}^{1, p(x)}(\Omega)$ and $W^{2, q(x)}(\Omega) \cap W_{0}^{1, q(x)}(\Omega)$, i.e., $E=\left(W^{2, p(x)}(\Omega) \cap W_{0}^{1, p(x)}(\Omega)\right) \times\left(W^{2, q(x)}(\Omega) \cap\right.$ $W_{0}^{1, q(x)}(\Omega)$ ), and $X$ denotes the Sobolev space $W^{2, p(x)}(\Omega) \cap W_{0}^{1, p(x)}(\Omega)$.

In recent years, the study of differential equations and variational problems with $p(x)$ growth conditions has been an interesting topic resulting from nonlinear electrorheological fluids (see [1]) and elastic mechanics (see [2]).

( 2013 Huang et al.; licensee Springer. This is an Open Access article distributed under the terms of the Creative Commons Attribution License (http://creativecommons.org/licenses/by/2.0), which permits unrestricted use, distribution, and reproduction in any medium, provided the original work is properly cited. 
Some authors considered elliptic systems (see [3-16]) which have been used in a wide range of applications. Existence and multiplicity results for elliptic systems involving variational structure have been extensively investigated.

In [3], Boccardo and Figueiredo studied the following boundary value problem involving the $(p, q)$-Laplacian:

$$
\left\{\begin{array}{l}
-\Delta_{\mathrm{p}} u=F_{u}(x, u, v) \\
-\Delta_{\mathrm{q}} u=F_{v}(x, u, v)
\end{array}\right.
$$

where $p$ and $q$ are real numbers larger than 1 .

In [4], applying the fibering method established by Pohozaev, Bozhkova and Mitidieri, the authors studied the existence of multiple solutions for quasilinear system involving a pair of $(p, q)$-Laplacian operators.

In [5], Chun Li and Chun-Lei Tang ensured the existence of three solutions for the problem

$$
\begin{cases}-\Delta_{p} u=\lambda F_{u}(x, u, v), & \text { in } \Omega, \\ -\Delta_{q} u=\lambda F_{v}(x, u, v), & \text { in } \Omega, \\ u=v=0, & \text { on } \partial \Omega,\end{cases}
$$

where $p>N, q>N$ and $F$ satisfies suitable assumptions.

In [6], Afrouzi and Heidarkhani studied the existence of three solutions for a class of Dirichlet quasilinear elliptic systems involving the $\left(p_{1}, \ldots, p_{n}\right)$-Laplacian. In [7], Jing-Jing Liu and Xia-Yang Shi proved the existence of multiple solutions for a quasilinear system involving a pair of $(p(x), q(x))$-Laplacian operators. In [8], Bin Ge and Ji-Hong Shen obtained multiple solutions for a class of differential inclusion systems involving the $(p(x), q(x))$ Laplacian.

Recently, Lin Li and Chun-Lei Tang (see [9]) considered the Navier boundary value problem involving the $(p, q)$-biharmonic systems

$$
\begin{cases}\Delta\left(|\Delta u|^{p-2} \Delta u\right)=\lambda F_{u}(x, u, v)+\mu G_{u}(x, u, v), & \text { in } \Omega, \\ \Delta\left(|\Delta u|^{q-2} \Delta u\right)=\lambda F_{v}(x, u, v)+\mu G_{v}(x, u, v), & \text { in } \Omega, \\ u=\Delta u=v=\Delta v=0, & \text { on } \partial \Omega,\end{cases}
$$

where $p>\max \left\{1, \frac{N}{2}\right\}, q>\max \left\{1, \frac{N}{2}\right\}$, and $F, G$ satisfy suitable assumptions.

The main result of this paper is the following theorem.

Theorem 1.1 Suppose that there exist two positive constants $C, d$ and two functions $\gamma(x), \beta(x) \in C(\bar{\Omega})$ with $1<\gamma^{-}<\gamma^{+}<p^{-}, 1<\beta^{-}<\beta^{+}<q^{-}$such that

(j1) $F(x, s, t) \geq 0$ for a.e. $x \in \Omega$ and all $(s, t) \in[0, d] \times[0, d]$;

(j2) $\exists p_{1}(x), q_{1}(x) \in C(\bar{\Omega})$ and $p^{+}<p_{1}^{-} \leq p_{1}(x)<p^{*}(x), q^{+}<q_{1}^{-} \leq q_{1}(x)<q^{*}(x)$ such that

$$
\limsup _{(\mathrm{s}, t) \rightarrow(0,0) x \in \Omega} \frac{F(x, s, t)}{|s|^{p_{1}(x)}+|t|^{q_{1}(x)}}<+\infty
$$

(j3) $|F(x, s, t)| \leq C\left(1+|s|^{\gamma(x)}+|t|^{\beta(x)}\right)$ for a.e. $x \in \Omega$ and all $(s, t) \in R \times R$; 
(j4) $F(x, 0,0)=0$ for a.e. $x \in \Omega$. Then there exist an open interval $\Lambda \subseteq[0,+\infty)$ and $a$ positive real number $r$ with the following property: for each $\lambda \in \Lambda$ and each function $G: \Omega \times R \times R \mapsto R$, measurable in $\Omega, C^{1}$ in $R \times R$ and satisfying

$$
\sup _{(x, s, t) \rightarrow(\Omega \times R \times R)} \frac{|G(x, s, t)|}{1+|s|^{p_{2}(x)}+|t|^{q_{2}(x)}}<\infty
$$

where $p_{2}, q_{2} \in C(\bar{\Omega})$ and $p_{2}(x)<p^{*}(x), q_{2}(x)<q^{*}(x)$ for all $x \in \bar{\Omega}$, there exists $\delta>0$ such that, for each $\mu \in[0, \delta]$, problem $(\mathrm{P})$ has at least three weak solutions whose norms in $\left(W^{2, p(x)}(\Omega) \cap W_{0}^{1, p(x)}(\Omega)\right) \times\left(W^{2, q(x)}(\Omega) \cap W_{0}^{1, q(x)}(\Omega)\right)$ are less than $r$.

The paper is organized as follows. In Section 2, we present some necessary preliminary knowledge about the Lebesgue and Sobolev spaces with variable exponents, and present Ricceri's three-critical-points theorem. In Section 3, we prove the main result.

\section{Preliminaries}

Assume that $\Omega$ is a bounded domain of $R^{N}(N \geq 1)$ with a smooth boundary $\partial \Omega$. Let

$$
\begin{aligned}
& C_{+}(\bar{\Omega})=\{h \mid h \in C(\bar{\Omega}), h(x)>1 \text { for all } x \in \bar{\Omega}\}, \\
& L_{+}^{\infty}(\Omega)=\left\{p \in L^{\infty}(\Omega): \underset{x \in \Omega}{\operatorname{essinf}} p(x)>1\right\} .
\end{aligned}
$$

For $p \in L_{+}^{\infty}(\Omega)$, set

$$
p^{-}=p^{-}(\Omega)=\underset{x \in \Omega}{\operatorname{essinf}} p(x), \quad p^{+}=p^{+}(\Omega)=\underset{x \in \Omega}{\operatorname{ess} \sup } p(x) .
$$

For $p \in L_{+}^{\infty}(\Omega)$, define

$$
L^{p(x)}(\Omega)=\left\{u \mid u: \Omega \rightarrow R \text { is measurable and } \int_{\Omega}|u|^{p(x)} d x<\infty\right\}
$$

with the norm

$$
\|u\|_{L^{p(x)}(\Omega)}=|u|_{p(x)}=\inf \left\{\lambda: \int_{\Omega}\left|\frac{u}{\lambda}\right|^{p(x)} d x \leq 1\right\}
$$

and

$$
W^{1, p(x)}(\Omega)=\left\{u \in L^{p(x)}(\Omega):|\nabla u| \in L^{p(x)}(\Omega)\right\}
$$

endowed with the norm

$$
\|u\|_{W^{1, p(x)}(\Omega)}=|u|_{p(x)}+|\nabla u|_{p(x)} .
$$

We denote by $W_{0}^{1, p(x)}(\Omega)$ the closure of $C_{0}^{\infty}(\Omega)$ in $W^{1, p(x)}(\Omega)$.

For the basic properties of the spaces $L^{p(x)}(\Omega), W^{1, p(x)}(\Omega)$ and $W_{0}^{1, p(x)}(\Omega)$, please refer to [17-20]. Now we recite some known results which will be used later. 
Proposition 2.1 (see [17])

(i) The spaces $L^{p(x)}(\Omega), W^{1, p(x)}(\Omega)$ and $W_{0}^{1, p(x)}(\Omega)$ are separable and reflexive Banach spaces;

(ii) If $q \in C_{+}(\bar{\Omega})$ and $q(x)<p^{*}(x)$ for any $x \in \bar{\Omega}$, then the imbedding from $W^{1, p(x)}(\Omega)$ to $L^{q(x)}(\Omega)$ is compact and continuous;

(iii) There is a constant $C>0$ such that $|u|_{p(x)} \leq C|\nabla u|_{p(x)} \forall u \in W_{0}^{1, p(x)}(\Omega)$.

By (iii) of Proposition 2.1, we know that $|\nabla u|_{p(x)}$ and $\|u\|$ are equivalent norms on $W_{0}^{1, p(x)}(\Omega)$. We use $|\nabla u|_{p(x)}$ to replace $\|u\|$ in the following discussion.

Proposition 2.2 (see [18]) Set $\rho(u)=\int_{\Omega}|u|^{p(x)} d x$ for $u, u_{k} \in L^{p(x)}(\Omega)$, we obtain

(1) For $u \neq 0,|u|_{p(x)}=\lambda \Leftrightarrow \rho\left(\frac{u}{\lambda}\right)=1$;

(2) $|u|_{p(x)}<1(=1 ;>1) \Leftrightarrow \rho\left(\frac{u}{\lambda}\right)<1(=1 ;>1)$;

(3) If $|u|_{p(x)}>1$, then $|u|_{p(x)}^{p^{-}} \leq \rho(u) \leq|u|_{p(x)}^{p^{+}}$;

(4) If $|u|_{p(x)}<1$, then $|u|_{p(x)}^{p^{+}} \leq \rho(u) \leq|u|_{p(x)}^{p^{-}}$;

(5) $\lim _{k \rightarrow \infty}\left|u_{k}\right|_{p(x)}=0 \Leftrightarrow \lim _{k \rightarrow \infty} \rho\left(u_{k}\right)=0$;

(6) $\left|u_{k}\right|_{p(x)} \rightarrow \infty \Leftrightarrow \rho\left(u_{k}\right) \rightarrow \infty$.

In this paper, the space $E$ is endowed with the following equivalent norm:

$$
\|(u, v)\|=\|u\|+\|v\|,
$$

where

$$
\|u\|=\inf \left\{\lambda>0: \int_{\Omega}\left|\frac{\Delta u}{\lambda}\right|^{p(x)} d x \leq 1\right\}, \quad\|v\|=\inf \left\{\mu>0: \int_{\Omega}\left|\frac{\Delta v}{\mu}\right|^{q(x)} d x \leq 1\right\} .
$$

Similar to Proposition 2.2, we obtain the following.

Proposition 2.3 Let $\phi(u)=\int_{\Omega}|\Delta u|^{p(x)} d x$ for $u, u_{k} \in W^{2, p(x)}(\Omega)$, we obtain:

(1) For $u \neq 0,\|u\|=\lambda \Leftrightarrow \phi\left(\frac{u}{\lambda}\right)=1$;

(2) $\|u\|<1(=1 ;>1) \Leftrightarrow \phi\left(\frac{u}{\lambda}\right)<1(=1 ;>1)$;

(3) If $\|u\|>1$, then $\|u\|^{p^{-}} \leq \phi(u) \leq\|u\|^{p^{+}}$;

(4) If $\|u\|<1$, then $\|u\|^{p^{+}} \leq \phi(u) \leq\|u\|^{p^{-}}$;

(5) $\lim _{k \rightarrow \infty}\left\|u_{k}\right\|=0 \Leftrightarrow \lim _{k \rightarrow \infty} \phi\left(u_{k}\right)=0$;

(6) $\left\|u_{k}\right\| \rightarrow \infty \Leftrightarrow \phi\left(u_{k}\right) \rightarrow \infty$.

Let $G(u)=\int_{\Omega} \frac{1}{p(x)}|\Delta u|^{p(x)} d x, u \in X$ and denote $L=G^{\prime}: X \rightarrow X^{*}$, then

$$
(L(u), v)=\int_{\Omega}|\Delta u|^{p(x)-2} \Delta u \Delta v d x \quad \forall u, v \in X .
$$

Proposition 2.4 (see [17])

(i) $L: X \rightarrow X^{*}$ is a continuous, bounded and strictly monotone operator;

(ii) $L$ is a mapping of type $\left(S_{+}\right)$, i.e., if $u_{n} \rightarrow u$ in $X$ and $\varlimsup_{\lim _{n \rightarrow \infty}}\left(\left(L\left(u_{n}\right)-L(u), u_{n}-u\right)\right) \leq 0$, then $u_{n} \rightarrow u$ in $X$;

(iii) $L: X \rightarrow X^{*}$ is a homeomorphism. 
Proposition 2.5 (see [21]) Let $X$ be a separable and reflexive real Banach space; $I \subseteq R$; let $\Phi: X \rightarrow R$ be a continuously Gâteaux differentiable and sequentially weakly lower semicontinuous functional whose Gâteaux derivative admits a continuous inverse on $X^{*}$; $J: X \rightarrow R$ is a continuously Gâteaux differentiable functional whose Gâteaux derivative is compact. In addition, $\Phi$ is bounded on each bounded subset of X. Assume that

$$
\lim _{\|u\| \rightarrow+\infty}(\Phi(u)+\lambda J(u))=+\infty
$$

for all $\lambda \in I \subseteq[0, \infty[$, and that there exists $\rho \in R$ such that

$$
\sup _{\lambda \in I} \inf _{u \in X}(\Phi(u)+\lambda(J(u)+\rho))<\inf _{u \in X} \sup _{\lambda \in I}(\Phi(u)+\lambda(J(u)+\rho)) .
$$

Then there exist a nonempty open set $A \subseteq I$ and a positive real number $r$ with the following property: for every $\lambda \in A$ and every $C^{1}$ functional $\Psi: X \rightarrow R$ with a compact derivative, there exists $\delta>0$ such that, for each $\mu \in[0, \delta]$, the equation

$$
\Phi^{\prime}(u)+\lambda J^{\prime}(u)+\mu \Psi^{\prime}(u)=0
$$

has at least three solutions in $X$ whose norms are less than $r$.

Proposition 2.6 (see [22]) Let $X$ be a nonempty set, and let $\Phi, J$ be two real functionals on $X$. Assume that there are $r>0$ and $x_{0}, x_{1} \in X$ such that

$$
\begin{aligned}
& \Phi\left(x_{0}\right)+J\left(x_{0}\right)=0, \quad \Phi\left(x_{1}\right)>r, \\
& \sup _{\left.\left.x \in \Phi^{-1}(]-\infty, r\right]\right)} J(x)<r \frac{J\left(x_{1}\right)}{\Phi\left(x_{1}\right)} .
\end{aligned}
$$

Then, for each $\rho$ satisfying

$$
\sup _{\left.\left.x \in \Phi^{-1}(]-\infty, r\right]\right)} J(x)<\rho<r \frac{J\left(x_{1}\right)}{\Phi\left(x_{1}\right)},
$$

one has

$$
\sup _{\lambda \geq 0} \inf _{x \in X}(\Phi(x)+\lambda(\rho-J(x)))<\inf _{x \in X} \sup _{\lambda \geq 0}(\Phi(x)+\lambda(\rho-J(x))) .
$$

\section{Proof of the main result}

Definition 3.1 A weak solution of problem $(\mathrm{P})$ is any $(u, v) \in E$ such that

$$
\begin{aligned}
& \int_{\Omega}\left(|\Delta u|^{p(x)-2} \Delta u \Delta \xi+|\Delta \nu|^{q(x)-2} \Delta v \Delta \eta\right) d x \\
& -\lambda \int_{\Omega}\left(F_{u} \xi+F_{\nu} \eta\right) d x-\mu \int_{\Omega}\left(G_{u} \xi+G_{\nu} \eta\right) d x=0
\end{aligned}
$$


for all $\forall(\xi, \eta) \in E$. We define the corresponding energy functional of problem (P) as

$$
\begin{aligned}
H(u, v) & =\Phi(u, v)+\lambda J(u, v)+\mu \Psi(u, v) \\
& =\int_{\Omega}\left(\frac{1}{p(x)}|\Delta u|^{p(x)}+\frac{1}{q(x)}|\Delta v|^{q(x)}\right) d x-\lambda \int_{\Omega} F(x, u, v) d x-\mu \int_{\Omega} G(x, u, v) d x,
\end{aligned}
$$

where

$$
\begin{aligned}
& \Phi(u, v)=\int_{\Omega}\left(\frac{1}{p(x)}|\Delta u|^{p(x)}+\frac{1}{q(x)}|\Delta v|^{q(x)}\right) d x, \\
& J(u, v)=-\int_{\Omega} F(x, u, v) d x ; \quad \Psi(u, v)=-\int_{\Omega} G(x, u, v) d x .
\end{aligned}
$$

Then $H(u, v)$ is a $C^{1}$ functional and the critical points of it are weak solutions of problem (P).

Proof of Theorem 1.1 Let $\Phi, J, \Psi$ as above. So, for each $u, v, \xi, \eta \in E$, one has

$$
\begin{aligned}
& \Phi^{\prime}(u, v)(\xi, \eta)=\int_{\Omega}\left(|\Delta u|^{p(x)-2} \Delta u \Delta \xi+|\Delta v|^{q(x)-2} \Delta v \Delta \eta\right) d x \\
& J^{\prime}(u, v)(\xi, \eta)=-\int_{\Omega} F_{u}(x, u, v) \xi d x-\int_{\Omega} F_{v}(x, u, v) \eta d x \\
& \Psi^{\prime}(u, v)(\xi, \eta)=-\int_{\Omega} G_{u}(x, u, v) \xi d x-\int_{\Omega} G_{v}(x, u, v) \eta d x .
\end{aligned}
$$

Therefore, the weak solutions of problem (P) are exactly the solutions of the equation

$$
\Phi^{\prime}(u, v)+\lambda J^{\prime}(u, v)+\mu \Psi^{\prime}(u, v)=0 .
$$

In view of Proposition 2.4 (or [17] for details), certainly, $\Phi$ is a continuously Gâteaux differentiable and sequentially weakly lower semi-continuous functional whose Gâteaux derivative admits a continuous inverse on $E^{*}$. Moreover, $J$ and $\Psi$ are continuously Gâteaux differentiable functionals whose Gâteaux derivatives are compact. Obviously, $\Phi$ is bounded on each bounded subset of $X$ under our assumptions.

By Proposition 2.3, set $G(u)=\int_{\Omega} \frac{1}{p(x)}|\Delta u|^{p(x)} d x$ just as before, then for $\|u\| \geq 1$,

$$
\frac{1}{p^{+}}\|u\|^{p^{-}} \leq G(u) \leq \frac{1}{p^{-}}\|u\|^{p^{+}}
$$

for $\|u\|<1$,

$$
\frac{1}{p^{+}}\|u\|^{p^{+}} \leq G(u) \leq \frac{1}{p^{-}}\|u\|^{p^{-}}
$$

Actually, for $\|u\|<1$, set $C_{0} \geq \frac{1}{p^{+}}\|u\|^{p^{-}}-\frac{1}{p^{+}}\|u\|^{p^{+}} \geq 0$, then we can obtain

$$
G(u)=\int_{\Omega} \frac{1}{p(x)}|\Delta u|^{p(x)} d x \geq \frac{1}{p^{+}}\|u\|^{p^{-}}-C_{0} .
$$


Hence we have

$$
G(u)=\int_{\Omega} \frac{1}{p(x)}|\Delta u|^{p(x)} d x \geq \frac{1}{p^{+}}\|u\|^{p^{-}}-C_{0}, \quad \forall u \in X
$$

So, there exists a constant $C_{1} \geq 0$ such that

$$
\begin{aligned}
\Phi(u, v) & =\int_{\Omega}\left(\frac{1}{p(x)}|\Delta u|^{p(x)}+\frac{1}{q(x)}|\Delta v|^{q(x)}\right) d x \\
& \geq \frac{1}{p^{+}}\|u\|^{p^{-}}+\frac{1}{q^{+}}\|u\|^{q^{-}}-C_{1}
\end{aligned}
$$

holds for any $(u, v) \in E$.

$$
\begin{aligned}
\lambda J(u, v) & =-\lambda \int_{\Omega} F(x, u, v) d x \\
& \geq-\lambda \int_{\Omega} C\left(1+|u|^{\gamma(x)}+|v|^{\beta(x)}\right) d x \\
& \geq-\lambda C\left(|\Omega|+|u|_{\gamma(x)}^{\gamma^{+}}+|u|_{\gamma(x)}^{\gamma^{-}}+|v|_{\beta(x)}^{\beta^{+}}+|v|_{\beta(x)}^{\beta^{-}}\right) \\
& \geq-C_{2}\left(1+|u|_{\gamma(x)}^{\gamma^{+}}+|v|_{\beta(x)}^{\beta^{+}}\right) \\
& \geq-C_{3}\left(1+\|u\|^{\gamma^{+}}+\|v\|^{\beta^{+}}\right)
\end{aligned}
$$

holds for any $(u, v) \in E$, where constants $C_{2} \geq 0, C_{3} \geq 0$. Here, by using conditions (j3) and (ii) of Proposition 2.1, combining the two inequalities above, we can obtain

$$
\Phi(u, v)+\lambda J(u, v) \geq \frac{1}{p^{+}}\|u\|^{p^{-}}+\frac{1}{q^{+}}\|v\|^{q^{-}}-C_{3}\left(1+\|u\|^{\gamma^{+}}+\|v\|^{\beta^{+}}\right)-C_{1} .
$$

Due to $\gamma^{+}<p^{-}, \beta^{+}<q^{-}$, we get

$$
\lim _{\|(u, v)\| \rightarrow+\infty}(\Phi(u, v)+\lambda J(u, v))=+\infty \quad \forall(u, v) \in E, \lambda \in[0, \infty) .
$$

Then assumption (2.1) of Proposition 2.5 is fulfilled.

Next, we derive that assumption (2.2) is also fulfilled. It is easy to verify the conditions of Proposition 2.6. Let $\left(u_{0}, v_{0}\right)=(0,0)$, we can easily have

$$
\Phi\left(u_{0}, v_{0}\right)=-J\left(u_{0}, v_{0}\right)=0
$$

Then there exist $\gamma>0$ and $\left(u_{1}, v_{1}\right) \in E$ such that $\Phi\left(u_{1}, v_{1}\right)>\gamma$ and (2.3) is satisfied.

There is a point $x^{0} \in \Omega$ since it is a nonempty bounded open set. Let $r_{2}>r_{1}>0$, put

$$
w(x)= \begin{cases}0, & x \in \Omega \backslash B\left(x^{0}, r_{2}\right), \\ \frac{d\left(3\left(l^{4}-r_{2}^{4}\right)-4\left(r_{1}+r_{2}\right)\left(l^{3}-r_{2}^{3}\right)+6 r_{1} r_{2}\left(l^{2}-r_{2}^{2}\right)\right)}{\left(r_{2}-r_{1}\right)\left(r_{1}+r_{2}\right)}, & x \in B\left(x^{0}, r_{2}\right) \backslash B\left(x^{0}, r_{1}\right), \\ d, & x \in B\left(x^{0}, r_{1}\right),\end{cases}
$$


where $B(x, r)$ is the open ball in $R^{N}$ of radius $r$ centered at $x$,

$$
l=\operatorname{dist}\left(x, x^{0}\right)=\sqrt{\sum_{i=1}^{N}\left(x_{i}-x_{i}^{0}\right)^{2}} .
$$

Let $\left(u_{1}(x), v_{1}(x)\right)=(w(x), w(x))$, then by (j1) we can derive that

$$
-J\left(u_{1}, v_{1}\right)=-J(w, w)=\int_{\Omega} F(x, w, w) d x>0 .
$$

From (j2), $\exists \eta \in[0,1], C_{1}>0$ such that

$$
\begin{aligned}
F(x, s, t) & <C_{1}\left(|s|^{p_{1}(x)}+|t|^{q_{1}(x)}\right) \\
& <C_{1}\left(|s|^{p_{1}^{-}}+|t|^{q_{1}^{-}}\right) \quad \forall(s, t) \in[-\eta, \eta] \times[-\eta, \eta] \text { a.e. } x \in \Omega .
\end{aligned}
$$

By (j3), there are nine positive real numbers $M_{i}(i=1,2, \ldots, 9)$ according to $|s|,|t|$ larger or smaller than $\eta$ and 1. For example, when $|s|>1,|t|<\eta$ some

$$
M_{i}=\sup _{|s|>1,|t|<\eta} \frac{C\left(1+|s|^{\gamma^{+}}+|s|^{\beta^{-}}\right)}{|s|^{-p_{1}^{-}}+|t|^{q_{1}^{-}}} .
$$

Set $M=\max \left\{C_{1}, M_{1}, \ldots, M_{9}\right\}$, then

$$
F(x, s, t)<M\left(|s|^{p_{1}^{-}}+|t|^{q_{1}^{-}}\right) \quad \forall(s, t) \in R \times R \text { a.e. } x \in \Omega \text {. }
$$

Hence, fix $\gamma$ such that $0<\gamma<1$. And for $\frac{1}{p^{+}}\|u\|^{p^{+}}+\frac{1}{q^{+}}\|v\|^{q^{+}} \leq \gamma<1$, by the Sobolev embedding theorem $\left(X \rightarrow L^{p_{1}^{-}}(\Omega)\right.$ is continuous), there exist suitable positive constants $C_{2}$ and $C_{3}$ such that

$$
\begin{aligned}
-J(u, v) & =\int_{\Omega} F(x, u, v) d x<M \int_{\Omega}\left(|u|^{p_{1}^{-}}+|v|^{q_{1}^{-}}\right) d x \\
& \leq C_{2}\left(\|u\|^{p_{1}^{-}}+\|v\|_{q_{1}^{-}}^{-}\right) \\
& \leq C_{3}\left(\gamma^{\frac{p_{1}^{-}}{P^{+}}}+\gamma^{\frac{q_{1}^{-}}{q^{+}}}\right) .
\end{aligned}
$$

Since $p_{1}^{-}>p^{+}, q_{1}^{-}>q^{+}$, we have

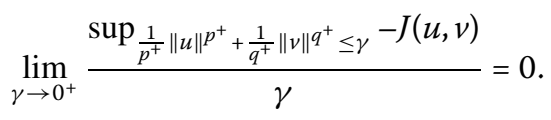

We choose $w(x) \in X$ as above such that $-J(w, w)>0$. Fix $\gamma_{0}$ such that $0<\gamma<\gamma_{0}<$ $\min \left\{\frac{1}{p^{+}}, \frac{1}{q^{+}}\right\} \cdot \min \left\{\|w\|^{p^{+}}+\|w\|^{q^{+}},\|w\|^{p^{-}}+\|w\|^{q^{-}}, 1\right\} \leq 1$. Then we divide the proof into two cases. 
(i) For $\|w\|<1$, by (3.2) we have

$$
\begin{aligned}
\Phi\left(u_{1}, v_{1}\right) & =\Phi(w, w) \\
& =\int_{\Omega}\left(\frac{1}{p(x)}|\Delta w|^{p(x)}+\frac{1}{q(x)}|\Delta w|^{q(x)}\right) d x \\
& \geq \min \left\{\frac{1}{p^{+}}, \frac{1}{q^{+}}\right\} \int_{\Omega}\left(|\Delta w|^{p(x)}+|\Delta w|^{q(x)}\right) d x \\
& \geq \min \left\{\frac{1}{p^{+}}, \frac{1}{q^{+}}\right\}\left(\|w\|^{p^{+}}+\|w\|^{q^{+}}\right) \\
& \geq \gamma_{0}>\gamma .
\end{aligned}
$$

By (3.3), we obtain

$$
\begin{aligned}
\sup _{\frac{1}{p^{+}}\|u\| p^{+}+\frac{1}{q^{+}}\|v\| q^{+} \leq \gamma}-J(u, v) & \leq \frac{\gamma}{2} \frac{-J\left(u_{1}, v_{1}\right)}{\max \left\{\frac{1}{p^{-}}, \frac{1}{q^{-}}\right\}\left(\|w\|^{p^{-}}+\|w\|^{q^{-}}\right)} \\
& \leq \frac{\gamma}{2} \frac{-J\left(u_{1}, v_{1}\right)}{\Phi\left(u_{1}, v_{1}\right)}<\gamma \frac{-J\left(u_{1}, v_{1}\right)}{\Phi\left(u_{1}, v_{1}\right)} .
\end{aligned}
$$

(ii) For $\|w\| \geq 1$, from (3.1) we get

$$
\begin{aligned}
\Phi\left(u_{1}, v_{1}\right) & =\Phi(w, w) \\
& =\int_{\Omega}\left(\frac{1}{p(x)}|\Delta w|^{p(x)}+\frac{1}{q(x)}|\Delta w|^{q(x)}\right) d x \\
& \geq \min \left\{\frac{1}{p^{+}}, \frac{1}{q^{+}}\right\} \int_{\Omega}\left(|\Delta w|^{p(x)}+|\Delta w|^{q(x)}\right) d x \\
& \geq \min \left\{\frac{1}{p^{+}}, \frac{1}{q^{+}}\right\}\left(\|w\|^{p^{-}}+\|w\|^{q^{-}}\right) \\
& \geq \gamma_{0}>\gamma .
\end{aligned}
$$

From (3.3), we have

$$
\begin{aligned}
\sup _{\frac{1}{p^{+}}\|u\| p^{p^{+}}+\frac{1}{q^{+}}\|v\| q^{+} \leq \gamma}-J(u, v) & \leq \frac{\gamma}{2} \frac{-J\left(u_{1}, v_{1}\right)}{\max \left\{\frac{1}{p^{-}}, \frac{1}{q^{-}}\right\}\left(\|w\| p^{+}+\|w\| q^{+}\right)} \\
& \leq \frac{\gamma}{2} \frac{-J\left(u_{1}, v_{1}\right)}{\Phi\left(u_{1}, v_{1}\right)}<\gamma \frac{-J\left(u_{1}, v_{1}\right)}{\Phi\left(u_{1}, v_{1}\right)} .
\end{aligned}
$$

For any $(u, v) \in \Phi^{-1}((-\infty, \gamma])$, we can obtain $\Phi(u, v)<\gamma$, i.e.,

$$
\Phi(u, v)=\int_{\Omega}\left(\frac{1}{p(x)}|\Delta u|^{p(x)}+\frac{1}{q(x)}|\Delta v|^{q(x)}\right) d x \leq \gamma .
$$

Then we can have

$$
\min \left\{\frac{1}{p^{+}}, \frac{1}{q^{+}}\right\} \int_{\Omega}\left(|\Delta u|^{p(x)}+|\Delta v|^{q(x)}\right) d x \leq \gamma .
$$


So,

$$
\int_{\Omega}\left(|\Delta u|^{p(x)}+|\Delta v|^{q(x)}\right) d x<\gamma \cdot \frac{1}{\min \left\{\frac{1}{p^{+}}, \frac{1}{q^{+}}\right\}}<\gamma_{0} \cdot \frac{1}{\min \left\{\frac{1}{p^{+}}, \frac{1}{q^{+}}\right\}}<1 .
$$

This inequality implies

$$
\int_{\Omega}|\Delta u|^{p(x)} d x<1, \quad \int_{\Omega}|\Delta v|^{q(x)} d x<1,
$$

i.e.,

$$
\|u\|<1, \quad\|v\|<1 .
$$

Therefore we have

$$
\frac{1}{p^{+}}\|u\|^{p^{+}}+\frac{1}{q^{+}}\|u\|^{q^{+}}<\int_{\Omega}\left(\frac{1}{p(x)}|\Delta u|^{p(x)}+\frac{1}{q(x)}|\Delta v|^{q(x)}\right) d x \leq \gamma .
$$

So, we can get that

$$
\Phi^{-1}((-\infty, \gamma]) \subset\left\{(u, v):(u, v) \in E, \frac{1}{p^{+}}\|u\|^{p^{+}}+\frac{1}{q^{+}}\|u\|^{q^{+}}<\gamma\right\} .
$$

Then

$$
\sup _{(u, v) \in \Phi^{-1}((-\infty, \gamma])}-J(u, v) \leq \sup _{\frac{1}{p^{+}}\|u\| p^{+}+\frac{1}{q^{+}}\|v\| \|^{+} \leq \gamma}-J(u, v)<\gamma \frac{-J\left(u_{1}, v_{1}\right)}{\Phi\left(u_{1}, v_{1}\right)},
$$

that is,

$$
\sup _{(u, v) \in \Phi^{-1}((-\infty, \gamma])}-J(u, v)<\gamma \frac{-J\left(u_{1}, v_{1}\right)}{\Phi\left(u_{1}, v_{1}\right)} .
$$

Hence we can find $\gamma>0, u_{1}=v_{1}=w$ and $\Phi(w, w) \leq \gamma$ satisfying (2.3). Also, we can find $\rho$ satisfying

$$
\sup _{(u, v) \in \Phi^{-1}((-\infty, \gamma])}-J(u, v)<\rho<\gamma \frac{-J\left(u_{1}, v_{1}\right)}{\Phi\left(u_{1}, v_{1}\right)} .
$$

Put $I=[0, \infty)$, moreover, $\Phi(u, v),-J(u, v)$ fulfil the assumption of Proposition 2.6. So, applying Proposition 2.6, we can easily get that (2.2) is fulfilled.

Thus, $\Phi, J$ and $\Psi$ fulfil all the assumptions of Proposition 2.5, and our conclusion follows from Proposition 2.5.

Remark Applying Theorem 2.1 in [23] to the proof of Theorem 1.1, an upper bound of the interval of parameters $\lambda$, for which $(\mathrm{P})$ has at least three weak solutions, is obtained. To be precise, in the conclusion of Theorem 1.1, one has

$$
\Lambda \subseteq\left[0, \frac{h \gamma}{\inf _{(u, v) \in \Phi^{-1}((-\infty, \gamma])} J(u, v)-\gamma \frac{J\left(u_{1}, v_{1}\right)}{\Phi\left(u_{1}, v_{1}\right)}}\right]
$$

for each $h>1$ and $\left(u_{1}, v_{1}\right)$ as in the proof of Theorem 1.1 (namely, $u_{1}=v_{1}=w$ ). 


\section{Competing interests}

The authors declare that they have no competing interests.

\section{Authors' contributions}

This paper is the result of joint work of all authors who contributed equally to the final version of this paper. All authors read and approved the final manuscript.

\section{Author details}

${ }^{1}$ School of Information \& Statistics, Guangxi University of Financial and Economics, Nanning, Guangxi 530003, China. ${ }^{2}$ Department of Construction and Information Engineering, Guangxi Modern Vocational Technology College, Hechi, Guangxi 547000, China. ${ }^{3}$ School of Mathematics and Statistics, Xuchang University, Xuchang, Henan 461000, China.

${ }^{4}$ School of Civil Engineering, Hebei University of Technology, Tianjin, 300130, China.

\section{Acknowledgements}

The authors would like to thank the editors and the referees for their valuable suggestions to improve the quality of this paper. This work was supported by the Scientific Research Project of Guangxi Education Department (No. 201204LX672).

\section{Received: 31 July 2013 Accepted: 4 September 2013 Published: 07 Nov 2013}

\section{References}

1. Ržuička, M: Electrorheological Fluids Modeling and Mathematical Theory. Springer, Berlin (2000)

2. Zhikov, W: Averaging of functionals of the calculus of variations and elasticity theory. Math. USSR, Izv. 9, 33-66 (1987)

3. Boccardo, L, Figueiredo, D: Some remarks on a system of quasilinear elliptic equations. Nonlinear Differ. Equ. Appl. 9, 309-323 (2002)

4. Bozhkova, Y, Mitidieri, E: Existence of multiple solutions for quasilinear systems via fibering method. J. Differ. Equ. 190, 239-267 (2003)

5. Li, C, Tang, CL: Three solutions for a class of quasilinear elliptic systems involving the ( $p, q)$-Laplacian. Nonlinear Anal. 69, 3322-3329 (2008)

6. Afrouzi, GA, Heidarkhani, S: Existence of three solutions for a class of Dirichlet quasilinear elliptic systems involving the $\left(p_{1}, \ldots, p_{n}\right)$-Laplacian. Nonlinear Anal. 70, 135-143 (2009)

7. Liu, JJ, Shi, XY: Existence of three solutions for a class of quasilinear elliptic systems involving the $(p(x), q(x))$-Laplacian. Nonlinear Anal. 71, 550-557 (2009)

8. Ge, B, Shen, JH: Multiple solutions for a class of differential inclusion system involving the $(p(x), q(x))$-Laplacian. Abstr Appl. Anal. 2012, Article ID 971243 (2012)

9. Li, L, Tang, CL: Existence of three solutions for (p, q)-biharmonic systems. Nonlinear Anal. 73, 796-805 (2010)

10. Drábek, P, Stavrakakis, NM, Zographopoulos, NB: Multiple nonsemitrivial solutions for quasilinear elliptic systems. Differ. Integral Equ. 16(12), 1519-1531 (2003)

11. Djellit, A, Tas, S: On some nonlinear elliptic systems. Nonlinear Anal. 59, 695-706 (2004)

12. Djellit, A, Tas, S: Quasilinear elliptic systems with critical Sobolev exponents in $R^{N}$. Nonlinear Anal. 66, 1485-1497 (2007)

13. Kristály, A: Existence of two non-trivial solutions for a class of quasilinear elliptic variational systems on strip-like domains. Proc. Edinb. Math. Soc. 48(2), 465-477 (2005)

14. Liu, Q: Existence of three solutions for $p(x)$-Laplacian equations. Nonlinear Anal. 68, 2119-2127 (2008)

15. Teramoto, T: On positive radial entire solutions of second-order quasilinear elliptic systems. J. Math. Anal. Appl. 282, 531-552 (2003)

16. Zhang, GQ, Liu, XP, Liu, SY: Remarks on a class of quasilinear elliptic systems involving the (p, q)-Laplacian. Electron. J. Differ. Equ. 2005(20), 1-10 (2005)

17. Fan, XL, Shen, JS, Zhao, D: Sobolev embedding theorems for spaces $W^{k, p(x)}(\Omega)$. J. Math. Anal. Appl. 262, 749-760 (2001)

18. Fan, $\mathrm{XL}$, Deng, SG: Remarks on Ricceri's variational principle and applications to the $p(x)$-Laplacian equations. Nonlinear Anal. 67, 3064-3075 (2007)

19. Fan, XL, Zhang, QH: Existence of solutions for $p(x)$-Laplacian Dirichlet problems. Nonlinear Anal. 52, 1843-1852 (2003)

20. Fan, $X L$, Zhao, D: On the spaces $L^{p(x)}(\Omega)$ and $W^{m, p(x)}(\Omega)$. J. Math. Anal. Appl. 263, 424-446 (2001)

21. Ricceri, B: A three critical points theorem revisited. Nonlinear Anal. 70, 3084-3089 (2009)

22. Ricceri, B: Existence of three solutions for a class of elliptic eigenvalue problem. Math. Comput. Model. 32, 1485-1494 (2000)

23. Bonanno, G: Some remarks on a three critical points theorem. Nonlinear Anal. 54, 651-665 (2003)

10.1186/1687-2770-2013-228

Cite this article as: Huang et al.: Existence of three solutions for a Navier boundary value problem involving the $(p(x), q(x))$-biharmonic. Boundary Value Problems 2013, 2013:228 